\title{
Enterocutaneous Fistula Developing In The Scar of an Appendicectomy With an Abdominal Tuberculosis: A Case Report
}

\author{
Arimino S*, Arijainalalao NH, Rakotomena D, Rajaonarivony T, Rakoto Ratsimba HN \\ Visceral Surgery, Hospital Universities - Joseph Ravoahangy Andrianavalona, Antananarivo Madagascar
}

\begin{abstract}
Varying forms of enterocutaneous fistula are described in the literature. In all cases, they have a functional, esthetic and even vital impact. The treatment may be complex because of the risk of a recurrence. We report the case of an enterocutaneous fistula developing in the short-term of an appendectomy at Mc Burney's point associated with an abdominal tuberculosis. The treatment of the tuberculosis has been essential to ensure the success of the surgery.
\end{abstract}

KEYWORDS: Appendicitis, Enterocutaneous Fistula, Pathology, Surgery, Tuberculosis

*Correspondence to: Sedera Arimino, Resident of General Surgery, Faculty de Medicine Antananarivo Madagascar, E-mail: sederaarimino02@gmail.com Received: October 14, 2019; Accepted: October 22, 2019; Published: October 25, 2019

\section{Introduction}

Morbidity and mortality of enterocutaneous fistula are estimated between 10 and 20\%. Most of them occur after digestive operation. Surgery may be considered according to the fistula output and its nutritional effect. Indeed, spontaneous healing is possible in $19.9 \%$ of cases [1-3] Persistent flow prompts to search for an underlying pathology [4]. We report the case of an enterocutaneous fistula that appeared a few days after a surgery associated with an abdominal tuberculosis.

\section{Case Report}

A 24 years old female patient presented to the hospital with an inexhaustible secretion coming out through a sinus opening on a surgical scar in her right iliac fossa. The disease has progressed for 2 years, starting a few days after an appendectomy at Mc Burney's point performed in a health center in a remote area. We didn't have access to the operating or the histopathology report. It was a serous fluid, sometimes stinky with an output of about $2 \mathrm{ml}$ per day maximum. She needed a daily dressing and underwent a second surgery in the same center 3 months later. She noted a recurrence after one week. On examination the patient had a Body Mass Index estimated to $17.5 \mathrm{~kg} /$ $\mathrm{m}^{2}$, a sinus discharging a serous liquid was found in the right iliac fossa, in an inflammatory scar [Figure 1].

There were no abdominal pain, mass or superficial lymph node palpable. There were no features of inflammation or White Blood Cells count disturbance. The abdominal sonography was normal and the abdominal tomography revealed a mesenteric calcification close to the caecum [Figure 2].

A median laparotomy was performed revealing a tubular structure without mesocolon visible, about $3 \mathrm{~cm}$ length, joining the caecum

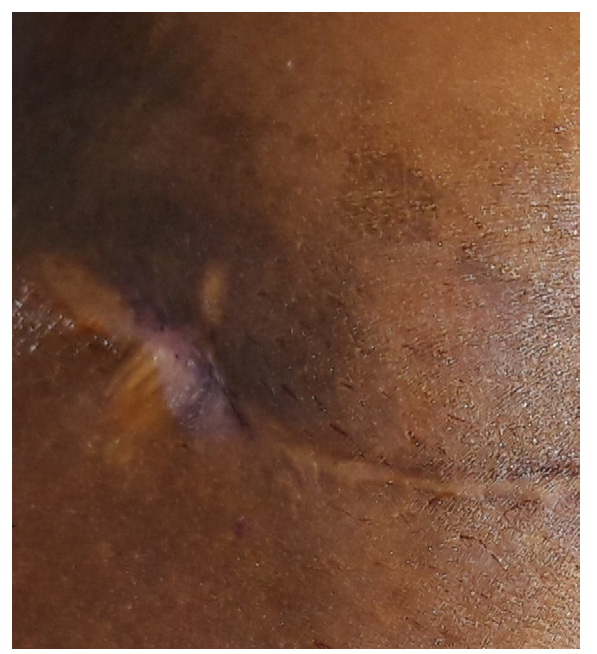

Figure 1: Sinus of the fistula in the scar of the appendicectomy at Mac Burney's point

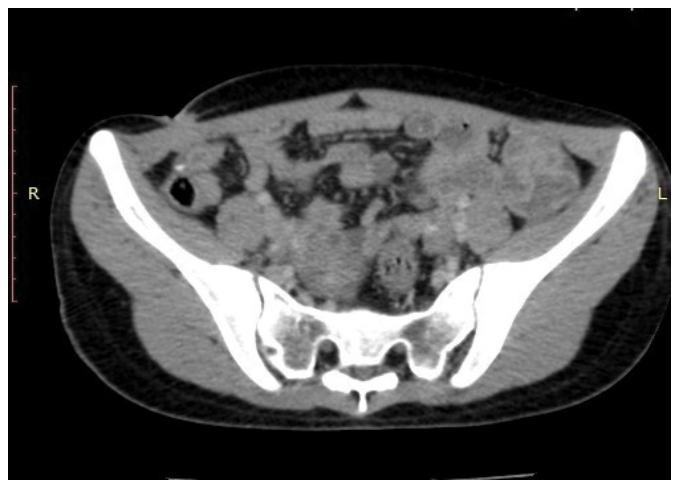

Figure 2: Computed Tomography section the mesenteric calcification next to the fistula zone. 
to the skin. In addition many white mesenteric nodules were found around, the largest one was about $2 \mathrm{~cm}$ [Figure 3].

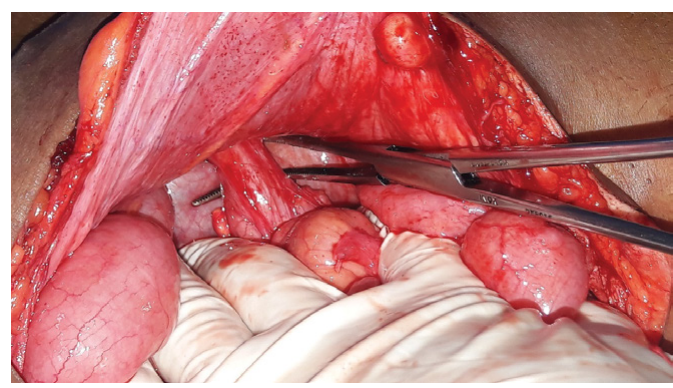

Figure 3: Fistular structure joining the caecum to the skin.

Excision of the fistula was done carrying the skin around. We made a biopsy of the nodule in the same time. Histopathological examination concluded to a tuberculosis infection, revealing cutaneous and subcutaneous tissue with suppurative granulomatous inflammation, many multinucleated giant cells Langhans-type and foci of caseous necrosis. The nodule was a calcified fibrous tissue. Fourdrug antitubercular therapy was started. After 7 months no recurrence was noted.

\section{Discussions}

Literatures recommend to perform percutaneous fistulography or Contrast Enhanced Computed Tomography for the diagnosis of enterocutanous fistula [2]. At one part, fistula appearing 30 days after a surgery or a radiotherapy are classified as spontaneous forms. Our case was noticed after a week, in favour of iatrogenic etiology. At the other part, a preexisting abdominal tuberculosis as an etiology of the appendicitis or even a risk factor of digestive fistula after surgery, seems conceivable. According to Singh, enterocutaneous fistula is a part of the rare abdominal tuberculosis complications (2.4\%). There are other intestinal diseases, except for the tuberculosis that may be mentioned as Crohn's disease, diverticulitis, cancer, radiation pathology. Performing a first biopsy on the edges of the fistula is recommended by some authors [2-4]. A low output fistula, that is to say with less than $100 \mathrm{ml}$ per day of discharge, doesn't need surgery apart from obstruction, infection, nutritional deterioration, or a persisting but low discharge $[2,3]$. In our case, surgery was performed at the request of the patient, in view of the borderline underweight, and the recurrence of the fistula less than 6 months after the second surgery. According to some authors, simple excision of the fistula without resection of the concerned intestinal portion may be a risk factor of recurrence [35]. Treating at the same time the underlying pathology plays also an important part to the management. The effectiveness of the surgery is estimated after 6 months [1].

\section{Conclusion}

Enterocutaneous fistula is a possible complication of a digestive surgery and of an abdominal tuberculosis. The success of the management needs the parallel treatment of the underlying pathology. This case also points to the need of an histopathological examination of all tissues removed from surgery.

\section{References}

1. Hollington P, Mawdsley J, Limb S, Gabe M, Forbes A et al (2004) An 11-Year experience of enterocutaneous fistula Br J Surg. 91: 1646-51.

2. Brenner AS, Baldin A, Molteni R, Ramos de Lima RF, Freitas LH, et al. (2017) Appendico-cutaneous fistula following hysterectomy first case report. J coloproctol 3: 157-9.

3. Singh O, Gupta S, Moses S, Jain DK Spntaneous (2009) Tubercular Enterocutaneous Fistula Developing in the Scar of a Surgery Done 14 Years Earlier. Saudi J Gastroenterol 15: 261-63

4. Kjellman T (1957) Appendiceal fistula and calculi: review of literature and a report of three cases. Acta Chir Scand 113:123-39.

5. Muthukumarassamy R, Raman Shankar R, Chandra Sarath S, Jagdish S (2005) Appendico-cutaneous fistula. Indian J Surg 67: 323-4. 